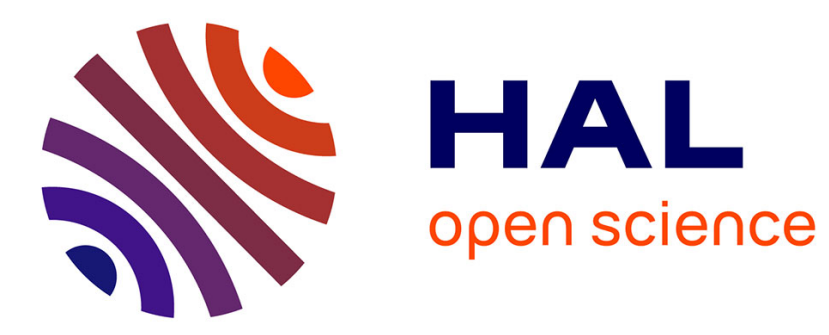

\title{
A polynomial time algorithm for determining the isomorphism of graphs of fixed genus
}

\author{
I.S. Filotti, J.N. Mayer
}

\section{To cite this version:}

I.S. Filotti, J.N. Mayer. A polynomial time algorithm for determining the isomorphism of graphs of fixed genus. [Research Report] RR-0008, INRIA. 1980. inria-00076553

\section{HAL Id: inria-00076553 \\ https://hal.inria.fr/inria-00076553}

Submitted on 24 May 2006

HAL is a multi-disciplinary open access archive for the deposit and dissemination of scientific research documents, whether they are published or not. The documents may come from teaching and research institutions in France or abroad, or from public or private research centers.
L'archive ouverte pluridisciplinaire HAL, est destinée au dépôt et à la diffusion de documents scientifiques de niveau recherche, publiés ou non, émanant des établissements d'enseignement et de recherche français ou étrangers, des laboratoires publics ou privés. 


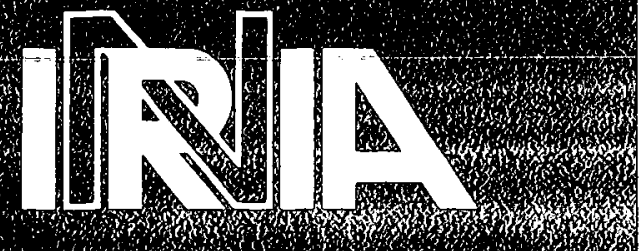

Institut 2 de Recherance

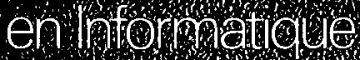
et en Atronatume

Domane ou volvear Rocquenouth BP105 78150 Le Chesna France

Tél. 9549020

\section{Rapports de Recherche}

$$
\mathrm{N}^{\mathrm{o}} 8
$$

A POLYNOMIAL-TIME ALGORITHM FOR DETERMINING THE ISOMORPHISM OF GRAPHS OF FIXED GENUS

Jack N. MAYER 
A POLYNOMIAL-TIME ALGORITHM FOR

DETERMINING THE ISOMORPHISM OF

GRAPHS OF FIXED GENUS

(working paper)

\author{
Ion S. FILOTTI ${ }^{\dagger}$ and Jack N. MAYER \\ Department of Computer Science Department of Mathematics \\ Columbia University, New York, N.Y. 10027
}

Résumé

Nous décrivons un algorithme en temps $p_{\gamma}\left(\alpha_{0}\right)$ pour déterminer 1'isomorphie de deux graphes de genre $\gamma$ à $\alpha_{0}$ sommets, où $\mathrm{p}_{\gamma}$ est un polynôme de degré linéaire en $\gamma$. La méthode repose sur : (1) un théorème de rigidité des graphes généralisant un théorème de Whitney et (2) 1'algorithme de Filotti et Miller pour plonger un graphe dans une surface compacte de genre $\gamma$.

\title{
Abstract
}

We describe an algorithm running in time $p_{\gamma}\left(\alpha_{0}\right)$ for determining the isomorphism of two graphs of genus $\gamma$ on $\alpha_{0}$ vertices, where $p_{\gamma}$ is a polynomial of degree linear in $\gamma$. The method is based on $:$ (1) a rigidity theorem for graphs generalizing a theorem of Whitney and (2) the algorithm of Filotti and Miller for embedding a graph in a compact surface of genus $\gamma$.

${ }^{+}$On visit at INRIA, 78150 LE CHESNAY, FRANCE. 
A Polynomial-time Algorithm for Determining the Isomorphism of Graphs of Fixed Genus (working paper)
I. S. Filotti*
Jack N. Mayer

Dept. of Computer Science

Dept. of Mathematics

Columbia University, New York, N.Y. 10027

\section{Introduction}

The isomorphism problem for graphs has been in recent years the object of a much research (see recent years the object of a much re complexity is still unknown. It is not known whether the problem is NP-complete, although it is NP, of course.

It is not known whether there exists a polynomialtime algorithm for it. Recently, Babai [Ba 79] has discussed probabilistic algorithms. For additional infusarion also information see aplications. Of the known algorisome practical applications. Of the known algorichms let us only quotarjan [Ho-Ta 72]. Weinberg's algorithm rums in quadratic time (in $\alpha_{0}$, the number of vertices of the graphs). Hopcroft and Tarjan's run in $O\left(\alpha_{0} \log x_{0}\right)$ and uses their powerful runs in of depth-first search. Both these algorithms apply only to planar (Weinberg's only to 3 -connected planar).graphs. They raly on a well known rigidity theorem of withney [Withney 32].

It was natural to try to extend these algorithms. to classes of non-planar graphs. This was not possible in the absence of better algorithms for embedding graphs in surfaces. Previous work by Filotti [Fi 78] and Filotii and Miller [Fi-Mi 79] was a necessary stepping stone to this end. This was a necessary stepping scorithm we shall present here for determining the isomorphism of graphs of genus $\gamma$. The algorithm runs in time $O\left(\alpha_{0}^{m+n}\right)$ for some positive constants an and $n$. This enlarges considerably the class of graphs For which there considerably the class of graphs

Weinberg's method relies on the fact that a planar 3-connected graph has only one embedding in the plane (actually two embeddings i $\vec{i}$ we count an embedding and its mirror image as different). This

*) On visit at INRIA, 78150 Le Chesnay, FRANCE. theorem is due to Wichney $\left.r_{\text {Withney }} 32\right]$. Weinberg shows how to canonically associace a code to planar embedding. From Withney's theorem it planar embedding. From that for planar 3-connected graphs the code depends only on the graph. To etablish the isomorphism of planar 3-connected graphs it suffices to compare the corresponding codes. This algorithm to compare the corresponding codas. polynomial in runs in time bounded by a quadratic polynomial in $\alpha_{0}$. Weinberg does not generalize his algorithm to arbitrary planar graphs. This is done by Hopcroft and Tarjan [Ho-Ta 72] who show how to construct a code for a graph from the codes of its 2-connected components and how to construct a code for a 2-connected graph from those associated to its 3-connected components. The latter depends on a decomposition due to Turte ? Tu 66]. This decomposition, and hence this part of the algorithm of Hopcroft and Tarjan, holds for arbitrary graphs. Hopcroft and Tarjan show how this decomposition can be achieved within time bounded by a polynomial can be (actually, within $\alpha_{0} \log \alpha_{0}$ steps). We can in $\alpha_{0}$ (actually, withise ourses to 3 -connected graphs.

For 3-connected graphs of higher genus the imediate generalization of Withney's theorem is false. Let $S_{\gamma}$ denote the compact surface of genus $\gamma$. A graph with only one embedding (up to mirror image) graph with only one embeddid. A graph $G$ is called in $S_{\gamma}$ will be called $\gamma$-rigid, where $\gamma(G)$ is the genus of G. Thus Withney's theorem asserts that planar 3 -connected graphs are $(0)-r i g i d$. In contrast, it
is easy to construct for every $\gamma>0$ an infinity of 3 -connected graphs that are not $y$-rigid.

Our algorithm relies on a generalization of Withney's rigidity theorem. Let $\mathrm{H}$ be a subgraph of $G$ and let $\mathrm{H}^{2}$ be an embedding of $\mathrm{H}$ in $S_{\gamma}$. The pair $\left(\mathrm{H}^{2}, \mathrm{G}\right)$ will be called rigid if there is just one (up to mirror image) embedding $\mathrm{G}^{2}$ of $\mathrm{G}$ in $\mathrm{S}_{\gamma}$ that extends $\mathrm{H}^{2}$. We shall also say that the extension extends $\left.\mathrm{H}^{2}, \mathrm{G}\right)$ is rigid. Theorem $i$ below, a generalization or Withney's theorem, will yield an important class of rigid extension problems. We then use techniques inspired from the embedding algorithms of Filotti and Miller (. $\mathrm{Fi}$ 78], Fi-Mi i9j) to construct our algorithm. 


\section{Notation, terminology and some basic results}

Notacion and cerminology is more or less as in Tutte [Tu 66] and Whice ' White 73] with the following additions. More details will be found in - Fi-Mi 79].

Graphs (i.e. CW-complexes of dimension 1) are written with superscript 1 (as in $\mathrm{H}^{1}, \mathrm{G}^{1}$, etc), embeddings (i.e. CW-complexes of dimension 2) are written with superscript 2. The superscript will be omitted when it is clear from the context. $G^{1}$ will in general denote the 1 -skeleton of $G^{2} . V(G)$, $E(G)$ and $F(G)$ denote respectively the set of vertices, edges and faces of $G$. Their cardinalities are respectively $\alpha_{0}(G), \alpha_{1}(G)$ and $\alpha_{2}(G)$. The Euler characteristic of $G^{l}$ is $x\left(G^{1}\right)=\alpha_{0}\left(G^{l}\right)-\alpha_{1}\left(G^{1}\right)$. The Euler characteristic of $G^{2}$ is $x\left(G^{2}\right)=\alpha_{0}\left(G^{2}\right)-\alpha_{1}\left(G^{2}\right)+\alpha_{2}\left(G^{2}\right)$. To any complex one can associate homology groups $\mathrm{H}_{i}\left(G^{j}\right)$

$(i=0,1, \ldots, j ; j=1,2)$. These are free abelian groups. The rank of $H_{i}\left(G^{j}\right)$ is denoted by $B_{i}\left(G^{j}\right)$. By the "alternating sum theorem", $\sum_{i=0}^{j}(-1)^{i} \alpha_{i}\left(G^{j}\right)=\sum_{i=0}^{j}(-1){ }_{i} B_{i}\left(G^{j}\right)$. Further, it can be shown that $B_{o}\left(G^{2}\right)=B_{2}\left(G^{2}\right) . B_{o}\left(G^{j}\right)$ equals the number of connected components of the underlying graph. For a graph, $B_{1}\left(G^{l}\right)$ equals its cyclomatic number, i.e. the number of independent cycles. From the alternating sum theorem, it follows that $\beta_{1}\left(G^{l}\right)=\alpha_{1}\left(G^{l}\right)-\alpha_{0}\left(G^{l}\right)+\beta_{a}\left(G^{l}\right)$. It can be shown that $x\left(G^{2}\right)$ is always even so that

$B_{1}\left(G^{2}\right)=2 \gamma\left(G^{2}\right)$ and $x\left(G^{2}\right)=2\left(\gamma\left(G^{2}\right)-B_{o}\left(G^{2}\right)\right)$ (Euler-Poincare formula). $Y\left(G^{2}\right)$ is called the genus of $G^{2}$. One says that $G^{2}$ is an embedding of $G^{l}$ in $S Y$, the surface of genus $\gamma$. For more derails see e.g. TFi-Mi 79] or standard textbooks on combinatorial topology. The genus of a graph $\mathrm{G}^{1}$ is $Y\left(G^{1}\right)=\min \left\{Y\left(G^{2}\right) / G^{2}=G^{1}\right\}$.

An embedding $G^{2}$ can be described by $a$ method due to, among others, Hefter and Edmonds (see e.g. (White 73]). Briefly, $G^{2}$ can be completely described by giving at each vertex of $G^{l}$ a cyclical ordering of the edges incident to it. To every embedding of $G$ one can associate a graph $G_{*}$ (depending on the embeding) as follows : associate a vertex to every face of the embedding and connect two vertices of $G_{\star}$ if the corresponding faces are adjacent(i.e. share an edge). $G_{\star}$ is called a (geometric) dual of $G$. There is a natural bijection between the edges of $G$ and those of $G_{m}$. Edmonds ([Ed 65]) has shown that an embedding is completely described by a geometric dual. In general the dual is not unique. This notion of dual muse not be confused with that of algebraic dual introduced by Withney, although the two are intimately connected. Further, the dual has a canonical embedding $G_{\star}^{2}$ of the same genus as $G^{2}$.

To a graph $G^{1}$ one associates a directed graph by associating to every edge of $\mathrm{G}^{\mathrm{l}}$ two arcs with opposite orientations. If the edge was e the two arcs will be denoted arbitrarily by $e$ and $e^{-1}$ and will be called the sides of the edge. A face of $G^{2}$ is a circuit in the digraph associated to $G^{l}$. If a face never traverses an edge or a vertex more than once it is called simpiy connected. An embedding all of whose faces are simply connected is called quasiolanar or simplicial. The first cerm was used in [Fi 78] and [Fi-Mi 79], but we prefer now the second. An edge or a vertex that is traversed more than once by the circuit associated to a face shall be called internal or repeated.

All our notions are combinatorical, but it will be quite often useful to use a geometrical language. This is motivated by the fact that to every embedding there is associated a topological space obtained as follows. To every face associate a copy of the unit disk of the complex plane. Map the oriented boundary of the disk onto the oriented circuit corresponding to the face. When composed with the morphism from the directed graph to the undirected graph that identifies opposite sides of the same edge and various copies of the same vertex, this results in a disk with a certain number of identifications on the boundary which we call the closed faced. The open face is simply the open disk before any identification. The closed faces are not necessarily simply connected and this is the source of many difficulcies.

If $\mathrm{H}^{1}$ is a subgraph of $\mathrm{K}^{1}$ we write $\mathrm{H}^{1} \subset \mathrm{K}^{1}, \mathrm{~K}^{2}$ is an extension of $\mathrm{H}^{2}$ if (a) $\mathrm{H}^{1} \subset \mathrm{K}^{1}$ and (b) the cyclical orientation at a vertex of $\mathrm{H}^{1}$ is the restriction of the cyclical orientation in $k^{2}$. An extension $\mathrm{K}^{2}>\mathrm{H}^{2}$ is conservative if $\gamma\left(\mathrm{K}^{2}\right)=\gamma\left(\mathrm{H}^{2}\right)$.

other notations are as follows :

$I_{Y}(G)$ denotes the class of embeddings of genus $Y$ of $G$.

$I(G)=\bigsqcup_{\gamma \geq 0} I_{\gamma}(G), I_{\gamma}(G)=\left|I_{\gamma}(G)\right|, I(G)=|I(G)|$.

$K_{\gamma}(G)$ denotes the class of all embeddings of șubgraphs of $G$.

$K(G)=\bigsqcup_{\gamma \geq 0} K_{Y}(G), x_{\gamma}(G)=\left|K_{Y}(G)\right|, K(G)=|K(G)|$.

$F_{\gamma}(G)$ denotes the class of all embetdings of $G$ that are frames (see section 3 ).

$F(G)=\bigcup_{Y \geq 0} F_{Y}(G), \phi_{\gamma}(G)=\left|F_{Y}(G)\right|, \phi(G)=|F(G)|$.

\section{The Rigidity Theorem}

Let $G^{2}$ be an embedding. A set of faces is said to contain a simplex if that simplex is contained in some subdivision of the faces. A non-contractible cycle on a surface is also called essential. i oraceilet is a pair $\left(f_{1}, f_{2}\right)$ of simply connected faces whose union contains an essential cycle. It is easy to see (using Jordan's theorem) that a bracelet must contain a pair $\left(s_{1}, s_{2}\right)$ ô disjoint sinplexes such that the cycle c consisting of two chains $c_{1}$ and $c_{2}$ joining $s_{1}$ to $s_{2}$ in $\hat{F}_{1}$ and $\hat{F}_{2}$ is essential. Such a pair is called a strap ot the bracelet. A bracelet may have more than one strap. A frome is a bracelet-free quasi-planar embedding.

Our goal in this section is to prove the Rigidity Theorem, Theorem 7 below.

The details of this proof will appear elsewhere [. Fi-May 80$]$. We shall only give a series of lemmas from which our theorem will follow.

Tutte in TTutte $66, p .112$ ] has introduced an operation which we shall call the expansion of a vertex. It consists of replacing a vertex $v$ of a 
graph by two vertices $v_{1}$ and $v_{2}$ connected by an edge. The edges incident to $v$ will be partitioned into two sets incident to $v_{1}$ and $v_{2}$ respectively. The remaining vertices and edges will stay unchanThe remaining vill be called ged. The expansion of a vertex witicion contains at proper if each member of the partition con least two members. A graph $x^{1}$ is called an expansion of $\mathrm{H}^{1}$ if it is obtained by a sequence expansions of vertices (notation : $A$ rex has valenexpansion is proper $\mathrm{K}^{2}$ of $\mathrm{K}^{1}$ induces a canonical $\mathrm{ce} \geq 3$. An embeding $\mathrm{H}^{2}$ of $\mathrm{H}^{1}$. Finally, $\mathrm{H}^{2}=\mathrm{K}^{2}$ if and only if $\mathrm{K}_{\star}^{\mathrm{l}}$ is an expansion of $\mathrm{H}_{\star}^{\mathrm{l}}$.

LEMMA 1. If $\mathrm{F}^{2}$ is a frame and $\mathrm{E}^{2} \subset \mathrm{G}^{2}$ then $\mathrm{G}^{2}$ is a frame.

LEMMA 2. Let $\mathrm{G}^{2} \mathrm{eH}^{2}$ be such that $\mathrm{H}_{\star}^{2}$ is a proper expansion of $\mathrm{G}_{*}^{2}$

(a) If $\mathrm{G}_{\star}^{\mathrm{l}}$ is 3 -connected then so is $\mathrm{H}_{\star}^{2}$.

(b) if $\mathrm{G}^{1}$ is simplicial (i.e. without loops or multiple edges) then so is $\mathrm{H}^{1}$.

LEMMA 3. Let $G^{2}$ be a frame of a 3-connected simplicial graph $G^{l}$. Then $G_{\star}^{1}$ is simplicial and 3-connected.

For any graph $G$ we denote by $C(G)$ the matroid of its cycles.

LEMA 4 (Withney). Let $G_{1}^{2}$ and $G_{2}^{2}$ be two planar embeddings of the same graph $G$. Then the natural edge bijection between $G_{1 *}^{l}$ and $G_{2 *}^{l}$ induces an isomorphism of $C\left(G_{1 *}^{1}\right)$ and $C\left(G_{2 \star}^{1}\right)$.

The following lemma is our generalization of the previous one.

LEMA 5. Let $F^{2}$ be a frame and let $F^{1} \subset G^{1}$. Let $G_{1}^{2}$ and $G_{2}^{2}$ be two embeddings of $G^{i}$ that are conservative extensions of $\square^{2}$. Then the nacural bijection between the edges of $\mathrm{G}_{1 \star}^{l}$ and of $\mathrm{G}_{2 *}^{1}$ induces an isomorphism of $C\left(G_{1 *}^{1}\right)$ and $C\left(G_{2 \star}^{1}\right)$.

IEMM 6 (Withney). Let $G$ and $H$ be 3-connected raphs. Let $f$ be a bijection between the edges of $G$ and those of $t$ that induces an isomorphism of $C(G)$ and those of $A$. Then $f$ induces an isomorphism of $G$ and $H$.

This leads us to the following important theorem.

THEOREM 7 (The Rigidity Theorem). Let $G^{1}$ be a -connected simplicial graph. Let $F^{2}$ be a frame and let $\gamma=\gamma\left(F^{2}\right)$. Let $G_{1}^{2}$ and $G_{2}^{2}$ be two conservative excensions of $E^{2}$ with $G^{1}$ as 1 -skeleton. Let $G_{i *}^{l}$ and $G_{2}^{1}$ be che corresponding duals. Then the canonical bijection between the edges of $G_{l_{*}}^{l}$ and $G_{2 *}^{l}$ induces an isomorphism of $G_{1 *}^{1}$ and $G_{2 \star}^{l}$. Hence $G_{1}^{2}$ and $G_{2}^{2}$ are identical. In particular, the extension $\left(F^{2}, G^{1}\right)$ is rigid.

Proof. $G_{1^{*}}^{l}$ and $G_{2 *}^{l}$ are proper expansions of $E_{\star}^{l}$. By Lemma $1, G_{1}^{2}$ and $G_{2}^{2}$ are Erames. By Lemma $3, G_{1 *}^{l}$ and $G_{2 *}^{1}$ are simplicial and 3 -connected. By Lemma 5 , $C\left(G_{1 *}^{l}\right)=C\left(G_{2 *}^{1}\right)$ under the natural bijection. Final- ly, $G_{1 *}^{1}=G_{2 *}^{1}$ under the natural bijection. Hence the two embeddings are the same by Edmond's characterization.

Remark. There is an easy algorithm that will construct the unique extension of $\mathrm{F}^{2}$ to $\mathrm{G}^{2}$. Namely, construct the $\mathrm{G}^{1}-\mathrm{F}^{1}$ that rests on $F^{1}$. We are select a chain of $\mathrm{G}^{1}-\mathrm{F}^{1}$ that rests on $\mathrm{F}$. We are sure by the Theorem that this chain can be embedded in a single way. Let $G_{2}^{2}$ be the resulting embedding. Proceed in the same manner with $G_{2}^{2}$. In this way sequence of embeddings is obtained terminating with $\mathrm{G}^{2}$. This algorithm clearly runs in time bounded by a polynomial in $\alpha_{0}\left(G^{l}\right)$. A closer analysis would result in a linear polynomial.

\section{Separation}

4.1. The proof of the Main Theorem in the

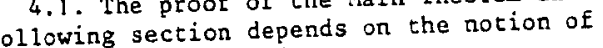
separation or removal.

Let $\mathrm{H}^{2} \in \mathrm{G}^{2}$ be a conservative extension and let $\mathrm{e}$ te to face $f$ of $\mathrm{H}^{2}$. If no edge be a chain incernal in $G^{2}$ we shall say that the interof $e$ is internal in $G^{2}$ we shall say been separated nal chain $e$ has been removed or has been seoarated in $\mathrm{G}^{2}$. Similary, if $\mathrm{v}$ is an internal vertex of that that is no longer internal in $G^{2}$ we shall say tha the internal vertex $v$ has been removed. Finally, if $\left(s_{1}, s_{2}\right)$ is a strap in $H^{2}$ but is no longer one in $\mathrm{G}^{2}$, we shall say that it has been removed or separated.

LEMMA 8. Let $\mathrm{H}^{2} \in \mathrm{G}^{2}$ be a conservative extension.

(a) If a chain e of $G^{2}$ is internal, then it is also internal in $\mathrm{H}^{2}$,

(b) If a vertex $v$ or $G^{2}$ is internal, then it is also internal in $\mathrm{H}^{2}$. in $\mathrm{H}^{2}$

(c) If $\left(s_{1}, s_{2}\right)$ is a strap in $G^{2}$ it is also one

Proof. Inmediate.

Thus an internal vertex that has been removed in some extensions cannot become internal in some further extension. Similar remarks hold for incernal edges and straps.

4.2. The tollowing version of Menger's theorem will be very useful. Let $S$ and $T$ be two disjoints subsets of $V\left(G^{l}\right)$. A set $X \in V\left(G^{l}\right)$ is said to subsets of $S$ and $T$ if after its deletion no composeparath of $S$ and one of $T$ (ci. e.g. 'Bo-Mu 75]). In particular, if $S$ is a singleton, removal of the vertex trivially separaces $S$ irom $T$.

LEMMA 9 (Menger). Let $S$ and $I$ be two disjoint sets of at least $k+1$ vertices. Let $c_{1}, c_{2}, \ldots, c_{k} T$. $(k \geq 1)$ be vertex-disjoint chains connecting $S$ to $T$. Then $S$ and $T$ are connected by $k+1$ vertex-disjoint chains if and only if no $k$-tuple $\left(v_{1}, v_{2}, \ldots, c_{k}\right.$ of from $\mathrm{T}$.

4.3 Let $\mathrm{H}^{2} \subset \mathrm{G}^{2}$ be a conservative extension. We shall associate to the extension a planar embedding 
called its planar representation, $P\left(H^{2}, G^{2}\right)$. To every Eace $F \in F\left(H^{2}\right)$ associate an undirected cycle in the plane, different faces receiving disjoint the plane, different faces rece then be embedded in these disks in exactly the same manner as in $G^{2}$. Figure 1 shows an example of such a representation.
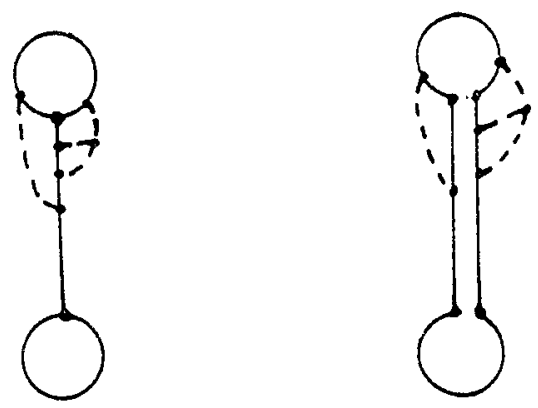

$\mathrm{B}^{2}$ is in solid lines

$\mathrm{G}^{2}-\mathrm{H}^{2}$ is in dotted lines

$P\left(H^{2}, G^{2}\right)$

\section{Figure 1}

By an abuse of language we denote the cycle that corresponds to $\mathrm{f}$ in $\mathrm{P}\left(\mathrm{H}^{2}, \mathrm{G}^{2}\right)$ by the same letter and the component corresponding to face $f$ by $f\left(\mathrm{G}^{2}\right)$. To the component cos of $\mathrm{H}^{2}$ there will correspond two edges in $f\left(G^{2}\right)$ called its sides. To every internal vertex of $\mathrm{H}^{2}$ there will correspond a number of ververtex of $\mathrm{a}^{2}$ there willed its comers. We call $\mathrm{P}\left(\mathrm{H}^{2}, \mathrm{H}^{2}\right)$ the planar representation of $\mathrm{H}^{2}$. If $\mathrm{H}^{2}$ $P\left(H^{2}, H^{2}\right)$ the planar represents simply of disjoint disks in the plane. Each edge of the boundary of disks in the plane. Each ed an eige of $\mathrm{H}^{2}$ and the two sides of an edge always occur in different disks.

4.4. Let again $\mathrm{H}^{2} \subset \mathrm{G}^{2}$ be a conservative extension, and let $f \in F\left(H^{2}\right)$ have an internal edge e. $f$ is then of the form $e e^{-1} b$ for some directed chains $a$ and $b$ of the directed graph associated to $\mathrm{H}^{\mathrm{l}}$. We shall $\mathrm{c} \exists 11 \mathrm{a}$ and $\mathrm{b}$ the components of the rim of $f$.

LEMMA 10. (a) $e$ is no longer internal in $G^{2}$ if and only if $f\left(G^{2}\right)$ has no articulation points in the interior of $\mathrm{e}$.

(b) if $G^{2}$ is quasi-planar, then $E\left(G^{2}\right)$ has no articulation points on $e$.

(c) if $e$ is no longer internal in $G^{2}$, then $f\left(G^{2}\right)$ contains two independant chains connecting the opposite sides of the rim.

(d) if $G^{2}$ is quasi-planar, then $f\left(G^{2}\right)$ contains two vertex-disjoint chains connecting the rims.

Proof. (a) and (b) follow from the fact that a planar embedding has an internal vertex if and only if the vertex is an articulation point. (c) and (d) follow from Lemma 9.
A pair of chains as in (c) or (d) is said to separate the chain $e$ in $f$.

We now turn to internal vertices.

LEXMA 11. Let $f \in F\left(H^{2}\right)$ and $v^{\prime}$ and $v^{\prime \prime}$ be two corners of the same vertex $v$. If $G^{2}$ has no repeated vertices then there exists a chain in $f\left(G^{2}\right)$ that separates $v^{\prime}$ and $v^{\prime \prime}$.

4.5 Extending slightly a notion of Tutte [Tu 66], let us call a pair $\left(s_{1}, s_{2}\right)$ of non-adjacent simplexes of $\mathrm{H}^{1}$ a hinge if its removal disconnect simplexes of be shown that in a planar graph the graph. It can be shown that in a planar if it a pair of simplexes is a hinge if and only the graph.

We now modify slightly the notion of strap. First, we shall allow the $s_{i}$ 's to be chains of edges, $s_{2}$ and $s_{2}$ being non-adjacent. Further, we shall insists that a strap be maximal, i.e. that no pair of non-adjacent chains $\left(s_{1}^{\prime}, s_{2}^{\prime}\right)$ be a strap if $s:>s_{i}$.

Consider now an extension $\mathrm{H}^{2} \subset \mathrm{G}^{2}$ with $\mathrm{H}^{2}$ quasiplanar. Let $\left(f_{1}, f_{2}\right)$ be a bracelec of $\mathrm{H}^{2}$. In $\mathrm{P}\left(\mathrm{H}^{2}\right)$, the embedding induced on a bracelet is, after identifying the common chains, of one of the following three types (Figure 2)

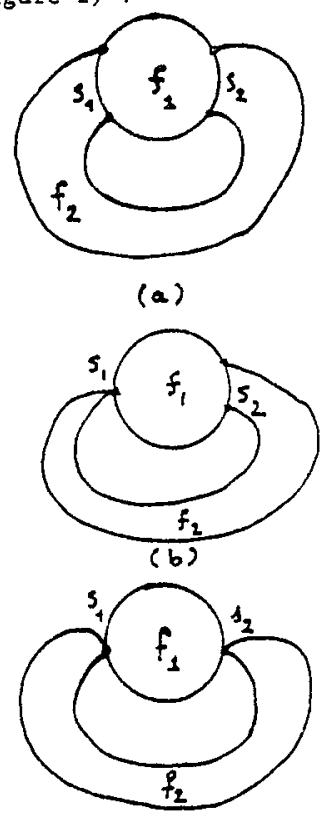

(c)

Figure 2

Define the rim of $\left(f_{1}, f_{2}\right)$ with respect to the strap as consisting of the vertices of (the boundary) $\xi_{1}$ and $f_{2}$ that are not iaterior to $s_{1}$ or to s? (a vertex being considered incerior to itself). In cases (a) and (b) the rim has two components. It has four in case (c).

We shall say that the $\operatorname{strap}\left(s_{1}, s_{2}\right)$ has been removed from $\left(\mathrm{F}_{1}, \mathrm{E}_{2}\right)$ if $\mathrm{G}^{2}$ has no pair $\left(\mathrm{s}_{1}^{1}, s_{2}^{1}\right)$ of non-adjacent chains and no pair $\left(E_{1}, F_{i}\right)$ of faces 
such that $\left(s_{1}^{1}, s_{2}^{1}\right) \subset\left(s_{1}, s_{2}\right),\left(f_{1}, f_{2}\right)=\left(f_{1}, f_{2}\right)$ and that $\left(s_{1}^{1}, s_{2}^{1}\right)$ is a strap of $\left(f_{1}, f_{1}^{1}\right)$.

A chain of $\mathrm{G}^{2}-\mathrm{H}^{2}$ all of whose edges are embedded in $f_{1}$ or $f_{2}$ will be said to be embedded in $f_{1} \cup f_{2}$.

LEMMA 12. Let $\mathrm{H}^{2}$ be quasi-planar and let $\mathrm{H}^{2} \subset \mathrm{G}^{2}$ be a conservative extension. Assume that $\mathrm{H}^{2}$ possesses a bracelet $\left(f_{1}, f_{2}\right)$ with strap $\left(s_{1}, s_{2}\right)$ that has been removed from $\left(f_{1}, f_{2}\right)$ in $G^{2}$. Then

(a) if both $s_{1}$ and $s_{2}$ are chains of at least one edge, then $G^{2}$ possesses three vertex-disjoint chains connecting the two components of the rim and embedded in $f_{1} \cup f_{2}$.

(b) if one only of the two components of $\left(s_{1}, s_{2}\right)$ is a chain of at least one edge, then $G^{2}$ possesses two vertex-disjoint chains connecting the two components of the rim and embedded in $f_{1} \cup f_{2}$.

(c) if $s_{1}$ and $s_{2}$ are both vertices, then they are separated by a chain embedded in either $F_{1}$ or $\mathrm{f}_{2}$.

Proof. The proof follows from Lemma 9 and the remark concerning the hinges of planar graphs.

4.6. The set $K\left(G^{2}\right)$ of embeddings of subgraphs of $G$ is partially ordered by $C$. This induces a partial ordering on any subset of $K\left(G^{l}\right)$. An antichain is a set of mutually incomparable embeddings under this ordering. It is maximal if no superset is an antichain.

Let $x, y \subset K(G)$. We shall say that $y$ dominates $x$ (notation : $X \leq Y$ ) if every embedding of $Y$ extends some embedding of $X$. A base for $X$ is an antichain dominated by $x$.

\section{The Main Theorem}

5.1 The Main Theorem that we shall prove in this section shows that a 3-connected graph cannot have too many Erames of genus $\gamma$. Moreover, they can be listed in polynomial time.

Recall that $\oplus_{\gamma}(G)$ denotes the number of embeddings of $G$ of genus $\gamma$ and that $i_{Y}(G)$ denotes the total number of embeddings of $G$ of genus $\gamma$.

THEOREM 13 (Main Theorem). For every genus $y \geq 0$ there exist polynomial $p_{y}$ and $q_{y}$ such that for any 3-connected graph $G$ :

(a) $\phi_{Y}(G) \leq p_{Y}\left(x_{O}(G)\right)$.

(b) the frames of $\mathrm{G}$ of genus $\gamma$ can be effectively listed in, time bounded by $q_{\gamma}\left(\alpha_{0}(G)\right)$.

Let us call a class of graphs $\gamma-\operatorname{semi-rigid}$ if there exists a polynomial $p_{y}$ such that

${ }^{1} \gamma(G) \leq p_{\gamma}\left(x_{O}(G)\right)$ for all graphs $G$ of the class. It follows from the Main Theorem that the class of graphs admitting only frames as embeddings in $S_{Y}$ is $\gamma$-semi-rigid.

To prove the Main Theorem, we shall construct a base $D(G)$ for $F(G)$. The procedure will be efficient i.e. will run in time bounded by a polynomial in $a_{0}(G)$. Consequently $|D(G)|$ is bounded by such a polynomial. By the Rigidity Theorem, it now follows that $G^{1}$ has no more than $|D(G)|$ frames of genus $Y$. Further, by the remark following the Rigidity Theorem, an extension of $\mathrm{F}^{2}$ to $\mathrm{G}^{1}$ can be constructed very efficiently. Thus, we have reduced the problem to that of the construction of $D(G)$.

To construct $D(G)$ we shall proceed along lines similar to those used in $\mathrm{rFi}^{2}$ 78] and $\mathrm{Fi}$-Mi 79 ?. Essentially, the method consists of starting with an embedding that is non-quasi-planar and to study the ways in which a quasi-planar embedding can extend it. We then proceed to remove the straps of the bracelets. More precisely, we sinall construct a sequence of antichains of embeddings $A(G) \leq B(G) \leq C(G) \leq D(G) \leq F(G)$, each member in the sequence being a base for the following one. The embeddings in $A(G)$ have only one face and are non-quasi-planar. The embeddings in $B(G)$ have no internal edges. $B(G)$ is obtained from $A(G)$ by removing the internal edges. $C(G)$ is obtained by removing the internal vertices of the embeddings of $B(G)$. Consequently, the embeddings of $C(G)$ are all quasi-planar. Finally, the straps are removed from the embeddings in $C(G)$ resulting in a base $D(G)$ of frames.

In what follows $\gamma \geq 0$ is fixed, $G$ is a fixed 3-connected graph and $\mathrm{T}$ is a fixed spanning tree of $G$.

\subsection{Constructetion_of $A(G)$}

For any set $S$ of edges of G-T, we let $T(S)$ be the subgraph constituted of $S$ together with all the paths of $T$ connecting two vertices $u$ and $v$ that are extremities of edges in $S . v(v)$ denotes the valence of vertex $v$.

LEMMA 14 ([Fi-Mi 79]). For any graph G :

(a) $\Sigma v(v)=2 \alpha_{I}(G)$, where the sum extends over a11 $v \in V(G)$.

(b) $\Sigma(v(v)-2)=2\left(B_{1}(G)-B_{0}(G)\right)$, where the sum extends over all $v \in V(G)$.

(c) $G$ has no more than $2\left(\beta_{l}(G)-\beta_{0}(G)\right)$ vertices of valence $\geq 3$.

(d) the total number of embedaings of $G$ does not exceed $\left(2 B_{1}(G)-2 B_{0}(G)+1\right) !$

(e) the total number of embeddings of $G$ does not exceed the number of embeddings of a bouquet of $\beta_{1}(G)$ circles.

Proof. (a) The sum on the left-hand side counts every edge cwice.

(b) $\Sigma(v(v)-2)=\Sigma v(v)-2 \alpha_{0}(G)=$

$=2 \alpha_{1}(G)-2 \alpha_{0}(G)=2\left(B_{1}(G)-B_{0}(G)\right)$.

(c) follows from (b) inmediately.

(d) $G$ has $\pi(v(v)-1)$ !. The produce concains $\Sigma(v(v)-1)$ factors of wich only $\Sigma(v(v)-2)$ are greater than 1 . The product cannot therefore excesd $(\varepsilon(\nu(v)-2)+1) !=\left(2 \beta_{1}(G)-2 g_{0}(G)+1\right) !$

(e) A bouquet of $\beta_{1}(G)$ circles has $\left(2 S_{1}(G)-1\right)$ ! embeddings. Since $\beta_{0}(G) \geq 1$ the results follows. A direct geometric proot is also possible.

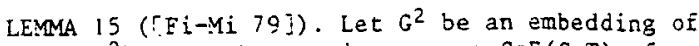
$G$ with $Y\left(G^{2}\right)=y$. There exiscs a set $S C E(G-T)$ of size $2 \gamma$ such that

(a) $\mathrm{G}^{2}$ induces on $\mathrm{H}=\mathrm{T}(\mathrm{S})$ an embedding $\mathrm{H}^{2}$ with $\gamma\left(\mathrm{H}^{2}\right)=\gamma$. 
(b) $\mathrm{H}$ is minimal with property (a).

(c) $\alpha_{2}\left(H^{2}\right)=\beta_{0}\left(H^{2}\right)$.

We let $A(G)=\left\{H^{2}, \mid H^{1}=T(S), S=E(G-T), \gamma\left(H^{2}\right)=\gamma\right\}$.

By Lemma $15, A(G)$ is a base for $I_{Y}(G)$. Clearly, $|A(G)| \leq\left(\begin{array}{c}\alpha_{1}(G) \\ 2 \gamma\end{array}\right) \cdot\left(2\left(2 \gamma-\beta_{0}(H)\right)+1\right) ! s\left(\begin{array}{c}\alpha_{1}(G) \\ 2 \gamma\end{array}\right)[(4 \gamma+1) !]$. Thus $|A(G)|=O\left(\alpha_{0}(G)^{i(\gamma)}\right)$.

For any graph its redustion is obtained by suppressing all vertices of valence 2 and replacing the two incident edges by a single one attached to their non-common extremity. Any embedding gives rise to exactly one of the reduction. The reductions of graphs in $A(G)$ have relatively few edges, as is seen in

LEMMA 16 ([Fi-Mi 79]). Let $H=T(S) \in A(G)$ and let $K$ be $i$ ts reduction. Then

(a) $\alpha_{0}(K) \leq 2\left(B_{1}(K)-B_{0}(K)\right) \leq 2(2 Y-1)$.

(b) $a_{1}(K) \leq 3\left(B_{1}\left(K^{2}\right)-B_{0}\left(K^{2}\right)\right) \leq 3(2 \gamma-1)$.

(c) It has at most $[2(2 \gamma-1)+1]$ ! embeddings.

Proof. $\beta_{1}(K)=\beta_{1}(H)=2 \gamma$ since the cyclomatic number equals the number of edges in the complement of a maximal tree. $B_{0}(K)=B_{O}(H) \leq 1$. By ment of a maximal $14(\mathrm{C}), x_{0}(K) \leq 2\left(\beta_{1}(K)-3_{0}(K)\right)=2\left(2 \gamma-\beta_{0}(K)\right)$ $\leq 2(2 \gamma-1)$. To obtain a bound on $\alpha_{1}(K)$ use Euler's formula and the fact that $\alpha_{2}\left(K^{2}\right)=\alpha_{2}\left(H^{2}\right)=\beta_{0}\left(H^{2}\right)=\beta_{0}\left(K^{2}\right)$ (by tema $15(\mathrm{c})$ ). This yields $\alpha_{1}\left(k^{2}\right)=\alpha_{0}\left(k^{2}\right)-\beta_{0}\left(k^{2}\right)+\beta_{1}\left(k^{2}\right) s$ $s 3\left(B_{1}\left(K^{2}\right)-B_{0}\left(R^{2}\right)\right) \leq 3(2 \gamma-1)$. Finally, (c) follows from Lema $14^{\circ}$ (d).

We shall now estimate the time required for the construction of $A(G)$. First the construction of $T$ can be done in $O\left(\alpha_{0} \log \alpha_{0}\right)$ steps (because, by Euler's formula, $\alpha_{1}$ is a linear function of $\alpha_{0}$ ), using for example Kruskal's greedy algorithm $\left.\left(r_{K r} 56\right]\right)$. There are $O\left(\alpha_{0}{ }^{2 \gamma}\right)$ choices for the set $S$. Each graph $H=T(S)$ admits at most $O\left(4 \gamma^{4} \gamma\right)$ embeddings. Thus the construction of $A(G)$ requires no more than $o\left(\alpha_{0}{ }^{2 \gamma}\right)$ steps (for $y \geq 1$, which is anyway the only case of real incerest).

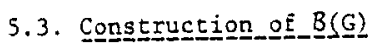

We shall construct a chain

$A(G)=B_{O}(G) \leq B_{1}(G) \leq \ldots \leq B_{r}(G)=B(G)$. Assume $H^{2} \in B_{i}(G)$ has been constructed. We shali construct from $\mathrm{H}^{2}$ a set of extensions that will belong to $B_{i+1}(G)$. If $H^{2}$ has no incernal edges then we let $\mathrm{H}^{2}$ be a nember of $B_{i+1}(G)$.

Assume that $\mathrm{H}^{2}$ has an internal chain e belonging to a face $f$. Using the notations of section 4.2 , let $a$ and $b$ be the rims of $f$. Let $L^{1}$ be the subgraph of $\mathrm{G}^{l}$ induced by the edges in $\left(\mathrm{G}^{l}-\mathrm{H}^{\mathrm{l}}\right) \cup\{\mathrm{e}\}$. By Lemma 10 , any frame extending $\mathrm{H}^{2}$ will contain two vertex-disjoint chains connecting the rims and embedded in $\vec{F}$. We would like to place in $B_{i+1}(G)$ all possible extensions obtained by augmenting $\mathrm{H}^{2}$ by two such chains. Unfortunately, this number is much too high. Observe however that if a chain $c$ has been embedded in $f$ then any other chain of $G^{1}-H^{1}$ that touches $c$ at an interior vertex will be also embedded in $f$. Therefore it suffices to specify the edges that begin and end the separating chains and then verify that such chains exist. To sumarize, $\mathrm{H}^{2}$ will generate a family or embeddings as follows: pick in rate a family or embeddings as follows : pick that have exactly one extremicy on a and two edges $e_{i}^{j}$ and ef in $L^{1}$ that have exactly one extremity on $b$. Using a standard max-flow-min-cut algorithm construct two disjoint chains $c_{1}$ and $c_{2}$ in $\mathrm{G}^{l}-\mathrm{H}^{1}$ connecting $\left\{e_{1}, e_{2}\right\}$ to $\left\{e_{i}, e_{2}^{\prime}\right\}$. If no such chains exist take the next choice. Let $\mathrm{K}^{2}$ be the embedding obtained by embedding $c_{1}$ and $c_{2}$ in $E$ and removing e. $B_{i+1}(G)$ will consist of all such embeddings.

Clearly, the chain $\left(B_{i}(G)\right)_{i \geq 0}$ beconmes stationary for $i$ greater than the number of internal edges of $\mathrm{H}^{2}$. We let $B(G)$ equal its limiting value.

The size of $B(G)$ is bounded by the number of choices for $\left\{e_{1}, e_{2}, e_{1}, e_{i}^{1}\right\}$ which is $O\left(a_{0}(G)^{4}\right)$. An upper bound on the running time of the max-rlow-mincut algorithm is $a_{0}(G)^{2}$. It is now easy to see that cut algorithm is $a_{0}(G)^{2}$. It is now easy to see that this algorithm run
a linear function.

For a graph $\mathrm{a}$ let $\mathrm{H}_{\text {red }}$ be its reduction. We shall now Estimate $\alpha_{0}\left(K_{r e d}^{L}\right)$ and $\alpha_{1}\left(K_{r e d}^{l}\right)$ Eor an embedding $K^{2} \in B(G)$. Let $H^{2} \in A(G)$ be such that $\mathrm{H}^{2} \in \mathrm{K}^{2}$. By Lenma $16, \alpha_{0}\left(H_{\text {red }}^{1}\right) \leq 2 \mu$ and $\alpha_{1}\left(H_{\text {red }}^{1}\right) \leq 3 \mu$ where we put $\mu=2 \gamma-1$. Every internal edge of $\mathrm{H}_{\mathrm{red}}^{1}$ gives rise in $k_{\text {red }}^{l}$ to at most two additional edges. Therefore $\alpha_{1}\left(K_{\text {red }}^{1}\right) \leq 6 \mathrm{u}$. Every time a chain is removed at most two new vertices are being created. Hence $\alpha_{0}\left(K_{\text {red }}^{l}\right) \leq 2 u+2.3 \mu=8 u$.

The number of internal vertices of $k_{r e d}^{2}$ does not exceed $8 \mu$ and the multiplicity of an internal vertex does not exceed bu.

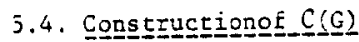

Let $K^{2} \in B(G)$. By Lemma- 11 , if $G^{2}$ is a $i r a m e$ of $G$, then any two co-facial cotners of the same vertex of $\mathrm{K}^{2}$ will be separated by a chain in $\mathrm{G}^{2}$. By Lemma 8 , it suffices to remove all the existing internal vertices and no new ones will be created in the process. Suppose that a face of $k^{2}$ has $r$ repeated vertices, each with $\mathrm{n}_{1}, \mathrm{n}_{2}, \ldots, \mathrm{n}_{\mathrm{r}}$ corners respectively. In $G^{2}$ any two consecutive corners of the same vertex must be separated by a chain. Therefore there can be at most $\Sigma n_{i}$ separating chains and at mose $2 \sum a_{i}$ different extremities.

Each embedding $R^{2} \leqslant B(G)$ will gererate embecidings $\mathrm{L}^{2}$ in $\mathrm{C}(\mathrm{C})$ as follows.

(a) For each face $f \in E\left(K^{2}\right)$ containing internal vertices and Eor each pair $\left(\mathrm{u}^{\prime}, \mathrm{u}^{\prime \prime}\right)$ of consecutive corners of the same vertex on $\mathrm{F}$, choose a pair $\left(v^{\prime}, v^{\prime \prime}\right)$ of corners of $f$ skew to $\left(u^{\prime}, u^{\prime \prime}\right)$. ( $\left(v^{\prime}, v^{\prime \prime}\right)$ will be candidate extremities of a chain separating $u^{\prime}$ and $u^{\prime \prime}$ and embedded in $f$.

(b) Find all assignnents of components or $G^{1}-K^{1}$ to each pair ( $\left.v^{\prime}, v^{\prime \prime}\right)$ of separating corners satisfying the following condition : if $C\left(v_{1}^{\prime}, v_{2}^{\prime \prime}\right)$ and 
$C\left(v_{2}^{\prime}, v_{2}^{\prime \prime}\right)$ are the components assigned to $\left(v_{1}^{\prime}, v_{1}^{\prime \prime}\right)$ and $\left(v_{2}^{\prime}, v_{2}^{\prime \prime}\right)$ respectively and if $\left(v_{1}^{\prime}, v_{1}^{\prime \prime}\right)$ and $\left(v_{2}^{\prime}, v_{2}^{\prime \prime}\right)$ are mutually skew then $\mathrm{C}\left(\mathrm{v}_{1}^{\prime}, \mathrm{v}_{1}^{\prime \prime}\right)=\mathrm{C}\left(\mathrm{v}_{2}^{\prime}, \mathrm{v}_{2}^{\prime \prime}\right)$.

(c) For each assignment in (b) construct in each component $C\left(v^{\prime}, v^{\prime \prime}\right)$ a chain connecting $v^{\prime}$ to $v^{\prime \prime}$.

(d) Embed the chains constructed at (c) in the corresponding faces.

(e) For each chain embedded in (d) retain only the portion between $f$ and the intersection point with another separating chain that is closest to the boundary of $f$.

$\mathrm{L}_{\text {red }}$ is obtained from $\mathrm{K}_{\text {red }}$ by adjoining at most $\sum v(v)$ separating chains. By Lemma 14 (b) we have $v \in \mathbb{K}^{1}$

$\Sigma \nu(v)=2 \mu+2 \alpha_{0}\left(K^{1}\right) \leq 2 \mu+2.8 \mu=18 \mu$, where as in

$5.4, \mu=2 \gamma-1$. The separating chains add at most $2.18 \mu=36 \mu$ vertices to those already in $k_{r e d}$ by perhaps subdivising an edge of $K_{\text {red }}$ and at most an additional $18^{2} \mu^{2}=324 \mu^{2}$ possible intersection points between the separating chains. However, since at (e) we retain on each chain only at most two edges and two vertices in addition to those on $\tilde{f}$, it follows that the separating chains add at most $72 \mu$ vertices. Hence $\alpha_{0}\left(\mathrm{~L}_{\mathrm{red}}^{\mathrm{L}}\right) \leq 72 \mu+8 \mu=80 \mu$. By a similar argument, it follows that $\alpha_{1}\left(L_{\text {red }}^{1}\right) \leq 96 \mu$. From Euler's formula we get $\alpha_{2}$ (Lred) $\leq 2-2 \gamma+\alpha_{1}\left(L_{r e d}^{l}\right) \leq 1+\mu+96 \mu=$ $=97 \mu+1 \leq 98 \mu$.

To evaluate the number of straps and bracelets, notice that every strap of $\mathrm{L}_{\text {red }}^{\mathrm{l}}$ must involve either an internal edge of $\mathrm{H}^{2}$ or an internal vertex of $\mathrm{K}^{2}$. Thus there cannot be more than $30 \mu$ straps.

The running time of this procedure is dominated by step (a). Step (a) requires the shoice of no more than $36 \mu$ vertices, hence $O\left(\alpha_{\circ}(G)^{36 \mu}\right)$ choices. Steps (b), (c), (d) and (e) require no more than $\alpha_{0}(G)^{3}$ steps.

\subsection{Constre}

This construction is based on Lemas 8 and 12 . By Lemma 8 once all straps have been removed no new ones have been created. By Lemma 12 the simplexes of a chain can be separated by one, two or three chains, depending on the nature of the strap. The number of straps of an embedding $L^{2} \in C(G)$ does not exceed $80 \mu(\mu=2 \gamma-1)$. To remove all straps no more than 240 u separating chains are needed. The construction is very similar to that of $B(G)$. We shall omit the details.

This completely concludes the proof. We have actually shown that the degree of the polynomial $\mathrm{P}_{\gamma}$ and $q_{\gamma}$ is linear in $\gamma$ as was stated in the Introduction (unforcunately this statement was omitted from the statement of Theorem 13). We have the following important

COROLLARY 17. There exists an algorithm that runs in time $O\left(\alpha_{0}(G)^{l(\gamma)}\right)$ for determining the iso- morphism of 3-connected graphs of genus $Y$ that admit only frames of genus $\gamma$, where $l$ is a linear function.

Proof. List of all frames of genus $Y$ of both graphs. Use then any standard labeling procedure (e.g. Weinberg's) to generate codes for the embeddings and then compare the codes.

\section{The isomorphism of 3-connected gragphs}

Let $v \in V\left(G^{l}\right)$. Giv will denote the graph obtained by replacing $v$ by $v(v)$ copies and making each adjecent to one of the $v(v)$ edges incident to $v$ in $G^{I}$. We say then that $v$ has been expioded. For $\mathrm{X} \subset \dot{V}\left(G^{1}\right)$ define $G(X$ inductively on $|\bar{X}|$ by $G \backslash(X \cup\{x\})=(G \backslash X) \backslash x$. An embedding $G^{2}$ of $G^{1}$ induces an embedding $G^{2} \backslash v$ on $G^{1} \backslash v$ restricting the cyclical orientations. For $\mathbb{Z} C E\left(G^{l}\right)$ we denote by $G^{l} \backslash \mathbb{X}$ the subgraph induced by $E\left(G^{l}\right)-\bar{X}$. For $e \in E\left(G^{l}\right)$ we write $G^{1} \backslash e$ instead of $G^{1} \backslash\{e\}$. $G^{2}$ induces an embedding $G^{2} U$ on $G^{l} U$ by restricting the fotations.

LEMMA 18 Let $G^{2}$ be an embedding of $G^{1}$.

(a) Assume $G^{l}$ is 2-connected. Then $v$ is an internal vertex of $G^{2}$ if and only if $\gamma\left(G^{2} \backslash v\right)<\gamma\left(G^{2}\right)$.

(b) Assume $G^{l}$ is 3 -connected. Then $(s, t)$ is $a$ strap of $G^{2}$ if and only if

(i) $\gamma\left(G^{2} \backslash s\right)=\gamma\left(G^{2}\right)=\gamma\left(G^{2} \backslash t\right)$,

(ii) $\gamma\left(G^{2} \backslash(s, c)\right)<\gamma\left(G^{2}\right)$.

(c) Let $\mathrm{G}^{\mathrm{i}}$ be 2 -connected. If $\mathrm{v}$ is an internal vertex of some embedding of genus $\gamma\left(G^{l}\right)$, then $\gamma\left(G^{l} \backslash v\right)<\gamma\left(G^{l}\right)$.

(d) Let $G^{l}$ be 3 -connected and let $(s, t)$ be a strap of an embedding of genus $Y\left(G^{l}\right)$ of $G^{i}$. Then

(i) $Y\left(G^{l} \backslash s\right)=Y\left(G^{l}\right)=\gamma\left(G^{l} \backslash t\right)$.

(ii) $Y\left(G^{I} \backslash(s, t)\right)<y\left(G^{l}\right)$.

The algorithm for deciding the isomorphism of two 3 -connected graphs $G_{1}$ and $G_{2}$ proceeds by bootstrapping. We assume an algorithm for genus $y^{-1}$ and lower. An algorithm for the case $Y=0$ is welt known ?Ho-Ta 72]. For simplicity, we may assume that the graphs are not non-isomorphic in "obvious: ways (e.g. they don't have the same degree sequence). The algorithm is then the rollowing.

(1) Check wether $Y\left(G_{1}\right)=Y=Y\left(G_{2}\right)$. This can be jone in time $O\left(\alpha_{O}(G) i(\gamma)\right.$ steps, where 1 is linear (?Fi-Mi 79]). If not the graphs are not isomorphic.

(2) Select all possible pairs $v_{1} \in V\left(G_{1}\right)$ and $v_{2} \in V\left(G_{2}\right)$ such that $y\left(G_{1} \backslash v_{1}\right)<\gamma\left(G_{1}\right)$ and $\gamma\left(G_{2} \backslash v_{2}\right)<\gamma\left(G_{2}\right)$ (i.e. such that $v_{i}$ is an internal vereex in some embedding of $G_{i}$ ). For each such pair execute scep 3 . When step 3 has been executed for ali pairs go to srep 4.

(3) Check wether $G_{1} \backslash v_{1} \sim G_{2} \backslash v_{2}$ using the isomorphism algorithm for genus $\because-1$. It can be shown that if $G_{1} \backslash v_{1} \sim G_{2} \backslash v_{2}$ then $G_{1} \sim G_{2}$. If $G_{1} \backslash v_{1} \sim G_{2} \backslash v_{2}$ stop. Else raturn to step (2).

(4) Select in all possible ways two pairs $\left(s_{1}, t_{1}\right)$ and $\left(s_{2}, t_{2}\right)$ co play the role of straps in $G_{1}$ and $G_{2}$ respectively. For each couple, check wether $\gamma\left(G_{1} \backslash\left(s_{1}, t_{1}\right)\right)<y\left(G_{1}\right)$ and $\gamma\left(G_{2} \backslash\left(s_{2}, t_{2}\right)\right)<\gamma\left(G_{2}\right)$. If this is so attach distinguishing tags to the vertices replacing $s_{i}$ and $\delta_{i}(i=1,2)$. Let $G_{i}^{\prime}$ and 
and $G_{2}^{\prime}$ be the resulting graphs. For each pair $\left(G_{1}^{1}, G_{2}^{j}\right)$ execute step (5). If all such pairs have been explored, go to step (6).

(5) Check, using the algorithm for genus $\gamma^{-1}$, wether $G_{1}^{\prime} \sim G_{2}^{\prime}$. It can be shown that if $G_{1}^{\prime} \sim G_{2}^{\prime}$ then $\mathrm{G}_{1} \sim \mathrm{G}_{2}$.

(6) At this stage, $G_{1}$ and $G_{2}$ admit only frames as embeddings of genus $\gamma$, hence $G_{1}$ and $G_{2}$ can be tested for isomorphism by the algorithm of Corollary 17.

This algorithm runs in time $p_{\gamma}\left(\alpha_{0}\left(G_{i}\right)\right)$ for some polynomial $P_{\gamma}$ of degree linear in $Y$.

\section{The general case}

The general case proceeds as in Cho-Ta 72.], as indicated in the Incroduction.

\section{References}

[Ba 79] L. Babai and L. Kucera, Canonical Labelling of Graphs in Linear Average Iime, Proc. of the 20-th Annual Symp. Foundations of Computer Science, Oct 29-31, 1979, San Juan, Puerto Rico, (published by the IEEE Computer Society).

r.Bo-Mu 75] J.A. Bondy and U.S.R. Murty, "Graph Theory with Applications", American Elsevier Publishing Company, New York, N.Y., 1976.

[Col 78] C.J. Colbourn, A Bibliography of the Graph Isomorphism Problem, Technical Report n ${ }^{\circ} 123$, Dept. of Computer Science, University of Toronto, 1978.

rEd 65] J. Edmonds, On the Surface Duality of Linear Graphs, J. Res. Natl. Bur. Standards-B. Mathematics and Mathematical Physics, 69.8, 1-2 (Jan.-June 1965), 121-123.

Fi 78] I.S. Filotti, An Algorithm for Imbedding Cubic Graphs in the Torus, J.C.S.S. 1980 (to appear). (A preliminary version of this paper was published in the Proc. Tenth Annual ACM Symp. Theory of Computing, San Diego, California, May 1980.

rFi-Mi 79] I.S Filotti and G. Miller, Efficient Determination of the Genus of a Graph (to appear). (A praliminary version of this paper was published in the Proc. Eleventh Annual ACM Symp. Theory of Computing, itlanta, Georgia, May 1979).

[Fi-May 80] A rigidity theorem for graph embeddings (unpublished).

rHo-Ta 72 ] J.E. Hopcroit and R.E. Tarjan, Isomorphism of Planar Graphs (Norking Paper) in "Complexity of Computer Computations" (Milier et al. eds.), Plenum Press, 1972.

rKr 56] 3.B. Kruskal, on the Shortest Spanning Subcree of a Graph and the Travelling Salesman Problem, Proc. Amer. Math. Soc., 7 (1956), 48-50.

Mi 77] G.L. Miller, Graph Isomorphism, General Remarks, Proc. 9-th ACM Symposium on the Theory of Computing, 143-150 (1977).
rRe-Cor 77] R.C. Read and D.G. Corneil, The Graph Isomorphism Disease, J. Graph Theory, I, 339-363 (1977).

PRe-Ni-De 77] Edward M. Reingold, Jurg Nievergelt and Narsingh Deo, "Combinatorial Algorithms : Theory and Practice", Prentice Hall, Englewood Cliffs, N.J.,1977.

rTu 66] W.T. Tutte, "Connectivity in Graphs", U. of Toronto Press, Toronto 1966.

Te 667 L. Weinberg, A Simple and Efficient Algorithm for Determining Isomorphism of Planar Triply Connected Graphs, IEEE Trans. On Circuit Theory, CT-13, 2 (1966), 142-148.

White 73? A. Whice, "Graphs, Groups and Surfaces", North-Holland Publishing Co., American Elsevier Co., New York, 1973.

[Whitney 32] H. Whitney, "Congruent Graphs and the Connectivity of Graphs", Amer. J. Math., $54(1932), 150-168$. 
